\title{
On the Coupled Forward and Backward Anisotropic Diffusion Scheme for Color Image Enhancement
}

\author{
Bogdan Smolka ${ }^{1 \star}$ and Konstantinos N. Plataniotis ${ }^{2}$ \\ 1 Department of Automatic Control, Silesian University of Technology \\ Akademicka 16 Str, 44-101 Gliwice, Poland \\ bsmolka@ia.polsl.gliwice.pl \\ 2 Edward S. Rogers Sr. Department of Electrical and Computer Engineering \\ University of Toronto, 10 King's College Road, Toronto, Canada \\ kostas@dsp.toronto.edu
}

\begin{abstract}
The use of low-level visual features to search and retrieve information in the multimedia databases has drawn much attention in the recent years $[1,2]$. Many of the existing techniques of image retrieval are based on image segmentation, which is a difficult task in many practical situations due to image noise and various compression artifacts. In this paper a novel approach to the problem of edge preserving smoothing, which allows to break an image into a set of homogeneous regions, is proposed and evaluated. The new algorithm is based on the combined forward and backward anisotropic diffusion with incorporated time dependent cooling process. This method is able to efficiently remove image noise while preserving and enhancing image edges. The proposed algorithm can be used as a first step of different techniques, which are based on color, shape and spatial location information, to search and retrieve information from multimedia databases.
\end{abstract}

\section{Anisotropic Diffusion}

Perona and Malik [3] formulate the anisotropic diffusion filter as a process that encourages intraregional smoothing, while inhibiting interregional denoising. The Perona-Malik (P-M) nonlinear diffusion equation is of the form [3-7] :

$$
\frac{\partial}{\partial t} \mathbf{I}(x, y, t)=\nabla[c(x, y, t) \nabla \mathbf{I}(x, y, t)]
$$

where $\mathbf{I}(x, y, t)$ denotes the color image pixel at position $(x, y), t$ refers to time or iteration step in the discrete case and $c(x, y, t)$ is a monotonically decreasing conductivity function, which is dependent on the image gradient magnitude

\footnotetext{
* This work was partially supported by KBN grant 8 T11E 01319 and NATO Col-
} laborative Linkage Grant LST. CLG. 977845 7T11A01021 
$c(x, y, t)=f(\| \nabla \mathbf{I}(x, y, t||)$ such as:

$$
\begin{aligned}
& c_{1}(x, y, t)=\exp \left\{-\left(\frac{\|\nabla \mathbf{I}(x, y, t)\|}{\beta}\right)^{2}\right\}, \\
& c_{2}(x, y, t)=\left\{1+\left(\frac{\| \nabla \mathbf{I}(x, y, t)||}{\beta}\right)^{2}\right\}^{-1}
\end{aligned}
$$

which were introduced in the original paper of Perona and Malik [3]. The parameter $\beta$ is a threshold parameter, which influences the anisotropic smoothing process. Using the notation $g=\|\nabla \mathbf{I}(x, y, t)\|, s=g / \beta$, where $\|\cdot\|$ denotes the vector norm, we obtain following formulas for the conductivity functions: $c_{1}(x, y, t)=\exp \left(-s^{2}\right), \quad c_{2}(x, y, t)=1 /\left(1+s^{2}\right)$. It is evident that the behavior of the anisotropic diffusion filter depends on the gradient threshold parameter $\beta$. To show the influence of $\beta$ it is helpful to define a flux function: $\Phi(x, y, t)=c(x, y, t)\|\nabla \mathbf{I}(x, y, t)\|$. With the flux function defined above, Eq. 1 can be rewritten as $\frac{\partial}{\partial t} \mathbf{I}(x, y, t)=\nabla \Phi(x, y, t)$. The flux functions $\Phi_{1}$ and $\Phi_{2}$ corresponding to conduction coefficients $c_{1}$ and $c_{2}$ are shown in Fig. 1.

As it is easy to notice in Fig. 1b, the flow increases with the gradient strength to reach a maximum and then decreases slowly to zero. This behavior implies that the diffusion process maintains homogenous regions since little smoothing flow is generated for low image gradients. In the same way, edges are preserved because the flow is small in regions where the image gradient is high.

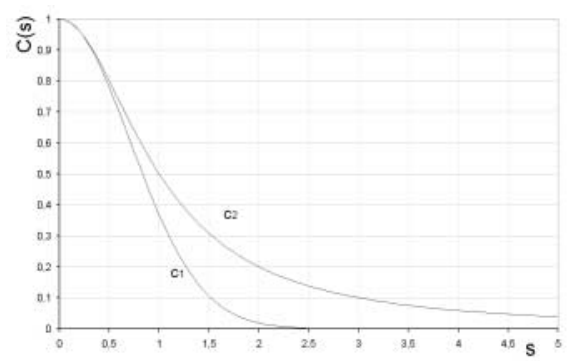

a)

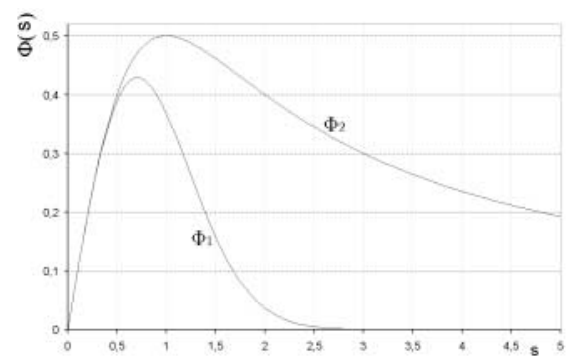

b)

Fig. 1. Dependence of the conductivity functions $c_{1}$ and $c_{2}(\mathbf{a})$, and the respective flux functions $\Phi_{1}$ and $\Phi_{2}(\mathbf{b})$, on the value of the normalized image gradient $s$

\section{Forward-and-Backward Diffusion}

The conductance coefficients in the P-M process are chosen to be a decreasing function of the signal gradient. This operation selectively smoothes regions 


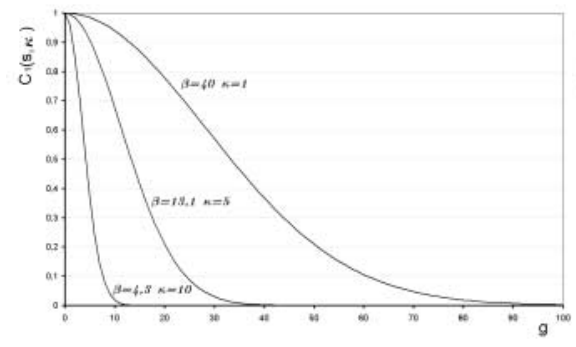

a)

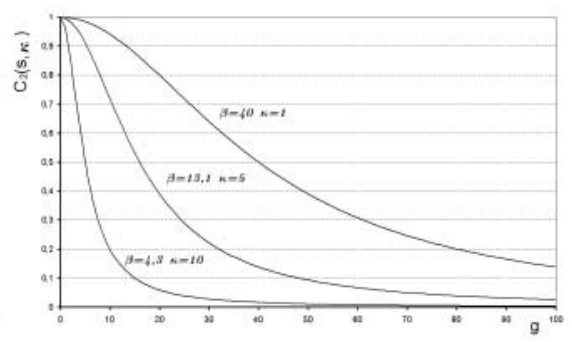

b)

Fig. 2. Dependence of the conductivity functions on the iteration step and the image gradient $g$ for the $c_{1}$ and $c_{2}$ conductivity functions, (forward diffusion, $\left.\beta_{1}=40, \gamma=0.8\right)$

that do not contain large gradients. In the Forward-and-Backward diffusion (FAB), a different approach is taken. Its goal is to emphasize the extrema, if they indeed represent singularities and do not come as a result of noise. As we want to emphasize large gradients, we would like to move "mass" from the lower part of a "slope" upwards. This process can be viewed as moving back in time along the scale space, or reversing the diffusion process. Mathematically, we can change the sign of the conductance coefficient to negative: $\frac{\partial}{\partial t} \mathbf{I}(x, y, t)=\nabla[-c(x, y, t) \nabla \mathbf{I}(x, y, t)], \quad c(x, y, t)>0$. However, we cannot simply use an inverse linear diffusion process, because it is highly unstable. Three major problems associated with the linear backward diffusion process must be addressed: explosive instability, noise amplification and oscillations.

One way to avoid instability explosion is to diminish the value of the inverse diffusion coefficient at high gradients. In this way, when the singularity exceeds a certain gradient threshold it does not continue to affect the process any longer. The diffusion process can be also terminated after a limited number of iterations. In order not to amplify noise, which after some pre-smoothing, can be regarded as having mainly medium to low gradients, the inverse diffusion force at low gradients should also be eliminated. The oscillations should be suppressed the moment they are introduced. For this, a forward diffusion force that smoothes low gradient regions can be introduced to the diffusion scheme.

The result of this analysis is that two forces of diffusion working simultaneously on the signal are needed - one backward force (at medium gradients, where singularities are expected), and the other, forward one, used for stabilizing oscillations and reducing noise. These two forces can actually be combined to one coupled forward-and-backward diffusion force with a conductance coefficient possessing both positive and negative values. In [8-10] a conductivity function 
that controls the FAB diffusion process has been proposed

$$
c_{F A B}(g)= \begin{cases}1-\left(g / k_{f}\right)^{n} & , \quad 0 \leq g \leq k_{f} \\ \alpha\left[\left(\left(g-k_{b}\right) / w\right)^{2 m}-1\right], & k_{b}-w \leq g \leq k_{b}+w \\ 0 & , \text { otherwise }\end{cases}
$$

where $g$ is an edge indicator (gradient magnitude or the value of the gradient convolved with the Gaussian smoothing operator), $k_{f}, k_{b}, w$ are design parameters and $\alpha=k_{f} /\left(2 k_{b}\right),\left(k_{f} \leq k_{b}\right)$ controls the ratio between the forward and backward diffusion. The dependence of such a defined conductance coefficient on the value of the gradient indicator is shown in Fig. $4 \mathrm{~b}$.

In the P-M equation, an "edge threshold" $\beta$ is the sole parameter, the FAB process described in [8-10] is modeled by a parameter which regulates forward force $k_{f}$, two parameters for the backward force (defined by $k_{b}$ and width $w$ ), and the relation between the strength of the backward and forward forces $\alpha$. Essentially $k_{f}$ is the limit of gradients to be smoothed out and is similar in nature to the role of $\beta$ parameter of the $\mathrm{P}-\mathrm{M}$ diffusion equation, whereas the $k_{b}$ and $w$ define the backward diffusion range.

In this study we propose two more natural conduction coefficients directly based on the P-M approach:

$$
c_{1_{F A B}}(s)=2 \exp \left(-s_{1}^{2}\right)-\exp \left(-s_{2}^{2}\right), \quad c_{2_{F A B}}(s)=\frac{2}{1+s_{1}^{2}}-\frac{1}{1+s_{2}^{2}} .
$$

The plots of the $c_{1_{F A B}}$ and $c_{2_{F A B}}$ diffusion coefficients are shown in Figs. 3, 4a. In the diffusion process smoothing is performed when the conductivity function is positive and sharpening takes place for negative conduction coefficient values.

\section{Cooling Down of the Diffusion Process}

Various modifications of the original diffusion scheme were attempted in order to overcome stability problems. Yet, most schemes still converge to a trivial solution (the average value of the image gray values) and therefore require the implementation of an appropriate stopping mechanism in practical image processing. In case of images contaminated by Gaussian noise, a common way of denoising is the usage of nonlinear cooling, which depends on the gradient, where large gradients cool faster and are preserved. In this study four simple time-dependent conduction coefficients were used:

$$
\begin{gathered}
c_{1}(g, t)=\exp \left[-\frac{g}{\beta(t)}\right]^{2}, \quad c_{2}(g, t)=\frac{1}{1+\left(\frac{g}{\beta(t)}\right)^{2}}, \\
c_{1_{F A B}}(s, t)=2 \exp \left[-\frac{g}{\beta_{1}(t)}\right]^{2}-\exp \left[-\frac{g}{\beta_{2}(t)}\right]^{2},
\end{gathered}
$$




$$
c_{2_{F A B}}(s, t)=\frac{2}{1+\left(\frac{g}{\beta_{1}(t)}\right)^{2}}-\frac{1}{1+\left(\frac{g}{\beta_{2}(t)}\right)^{2}} .
$$

where $g=\|\nabla \mathbf{I}(x, y, t)\|$ is the $L_{1}$ or $L_{2}$ norm of the color image vector in the RGB space, $\beta_{i}(t+1)=\beta_{i}(t) \cdot \gamma, \gamma \in(0,1], \beta_{i}(1)$ is the starting parameter, $i=1,2, \beta_{1}(t)<\beta_{2}(t)$.

The scheme depends only on two (in case of forward or backward diffusion) or three (in case of FAB diffusion) parameters: initial values of starting $\beta_{i}$ parameters and the cooling rate $\gamma$. Setting $\gamma$ to 1 means, that there is no cooling in the system. As $\gamma$ decreases, the cooling is faster, less noise is being filtered but edges are better preserved. Figures $3 \mathrm{a}$ and $3 \mathrm{~b}$ illustrate the dependence of diffusion coefficients $c_{1}(s, t)$ and $c_{2}(s, t)$ on iteration step $t$. The behaviour of the diffusion coefficients $c_{1_{F A B}}(s, t)$ and $c_{1_{F A B}}(s, t)$ are compared in Fig. 4.

If the cooling coefficient $\gamma$ is lower than 1 , then the gradient threshold $\beta(t)$ decreases with time, allowing lower and lower gradients to take part in the smoothing process. As time advances, only smoother and smoother regions are being filtered, whereas large gradients can get enhanced due to local inverse diffusion. The scheme converges to a steady state for $\beta \rightarrow 0$, which means that no diffusion is taking place.

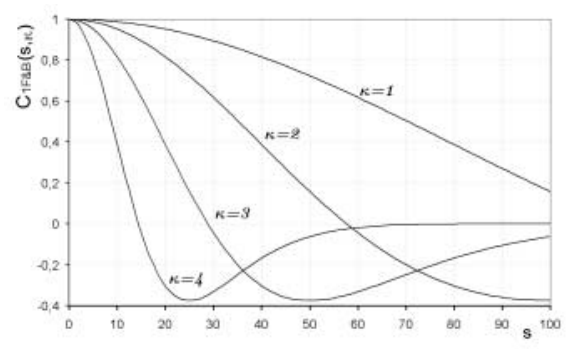

a)

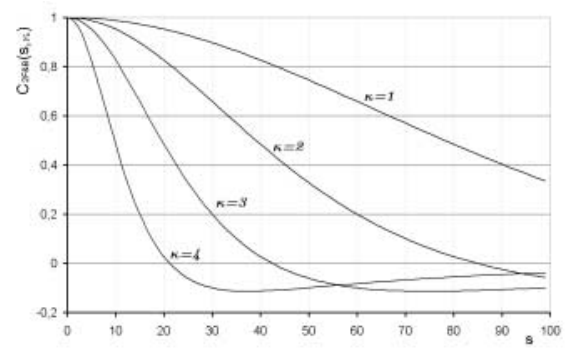

b)

Fig. 3. Dependence of the conductivity functions on the iteration number $\kappa$ and the normalized image gradient $s$ for the $c_{1 F A B}$ and $c_{2 F A B}$ conductivity functions, (forward and backward diffusion), for $\beta_{1}(1)=40, \beta_{2}(1)=80$ and $\gamma=0.5$. Note, that because of low $\gamma=0.5$ already in the second iteration, the conductivity functions have negative values for large enough gradients

\section{Experimentations and Results}

In this paper a novel approach to the problem of edge preserving smoothing is proposed. The experiments revealed that better results of noise suppression 


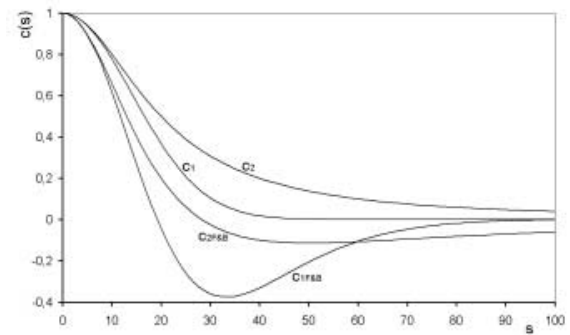

a)

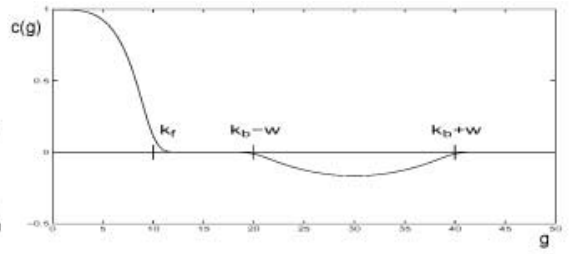

b)

Fig. 4. a) Comparison of the shape of the proposed forward and backward diffusion conductivity functions, b) the forward and backward conductivity function proposed in [8-10]

using the FAB scheme were achieved using the conductivity function $c_{2}$ from the original P-M approach (7). This is due to the shape of the coupled forward and backward conductivity shown in Fig. 3b, which allows more efective image sharpening.

The efficiency of the proposed technique is presented in Fig. 5, where two images are enhanced using the purely backward and FAB anisotropic techniques. The ability of the new algorithm to filter out noise and sharpen the color images is shown in Fig. 6, where the original color images were contaminated with Gaussian noise $(\sigma=30)$ and restored with the FAB anisotropic diffusion scheme. Another examples of the new filter efficiency are shown in Figs. 8 and 9.

The results confirm excellent performance of the new method, which could be used for the enhancement of noisy images in different techniques which are based on color, shape and spatial location information to search and retrieve information from multimedia databases.

\section{References}

1. Special Issue on Content Based Image Retrieval, IEEE PAMI, Vol. 8, No. 8, August, 1996

2. Special Issue on content based image retrieval, IEEE Computer Vol. 28, No. 9, September, 1995

3. P. Perona, J. Malik, Scale space and edge detection using anisotropic diffusion, IEEE PAMI, Vol. 12, No. 7 pp. 629-639, July 1990

4. R. Whitaker, G. Gerig, Vector-Valued Diffusion, Ed. B. M. ter Haar Romeny, "Geometry-Driven Diffusion in Computer Vision", Kluwer Academic Press, pp. 93-134, 1994 
5. M. J. Black, G. Sapiro, D. H. Marimont and D. Heeger, Robust anisotropic diffusion, IEEE Transactions on Image Processing, Vol. 7 (3), pp. 421-432, March 1998

6. J. Weickert, "Anisotropic Diffusion in Image Processing", Stuttgart-Germany: Teubner-Verlag, 1998

7. P. Blomgren, T. Chan, Color TV: Total variation methods for restoration of vector valued images, IEEE Transactions on Image Processing, March 1998, Special issue on Geometry Driven Diffusion and PDEs in Image Processing

8. G. Gilboa, Y. Zeevi, N. Sochen, Anisotropic selective inverse diffusion for signal enhancement in the presence of noise, Proc. IEEE ICASSP-2000, Istanbul, Turkey, vol. I, pp. 211-224, June 2000

9. G. Gilboa, Y. Zeevi, N. Sochen, Resolution enhancement by forward-and-backward nonlinear diffusion process, Nonlinear Signal and Image Processing, Baltimore, Maryland, June 2001

10. G. Gilboa, Y. Zeevi, N. Sochen, Signal and image enhancement by a generalized forward-and-backward adaptive diffusion process, EUSIPCO-2000, Tampere, Finland, September 2000 

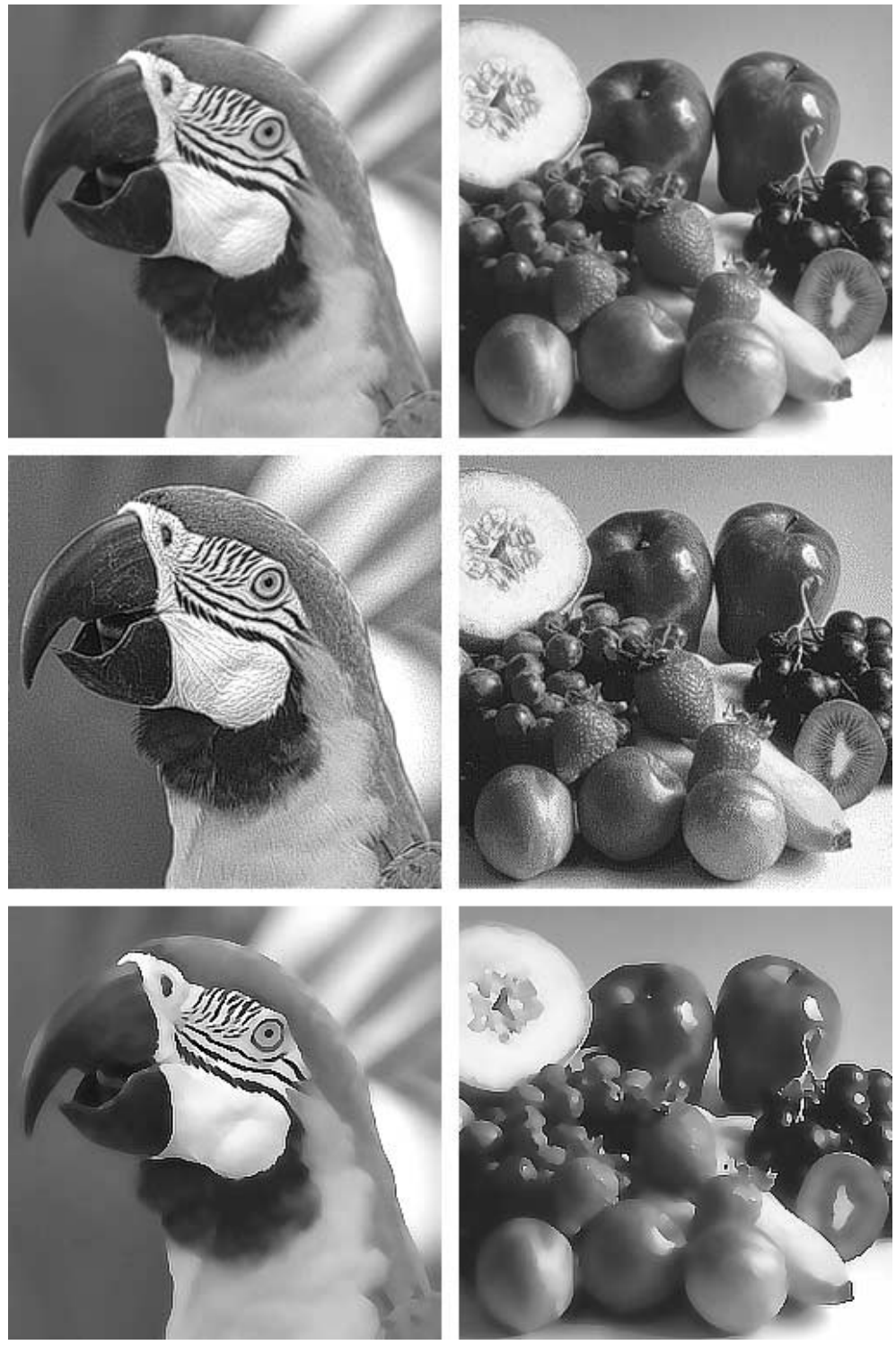

Fig. 5. Illustration of the new combined forward and backward anisotropic diffusion scheme. Top: color test images, below images enhanced with the pure backward diffusion and at the bottom images enhanced with the FAB diffusion scheme presented in the paper 

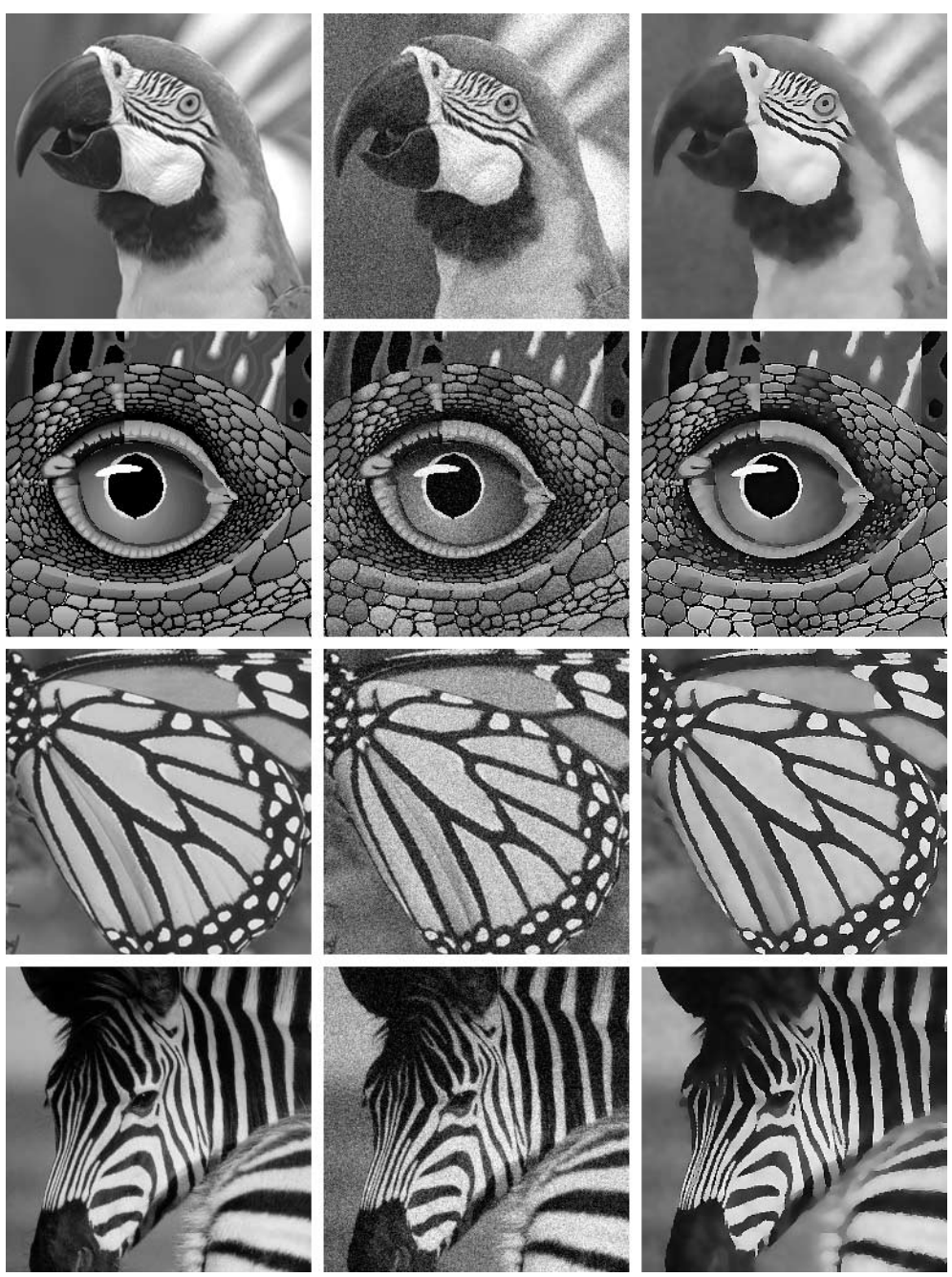

Fig. 6. Efectivness of the new coupled forward and backward anisotropic diffusion scheme. Lef column: color test images, in the center images contaminated with additive Gaussian noise $(\sigma=30$ added independently on each RGB channel), to the right images enhanced with the new FAB anisotropic diffusion scheme (7) 

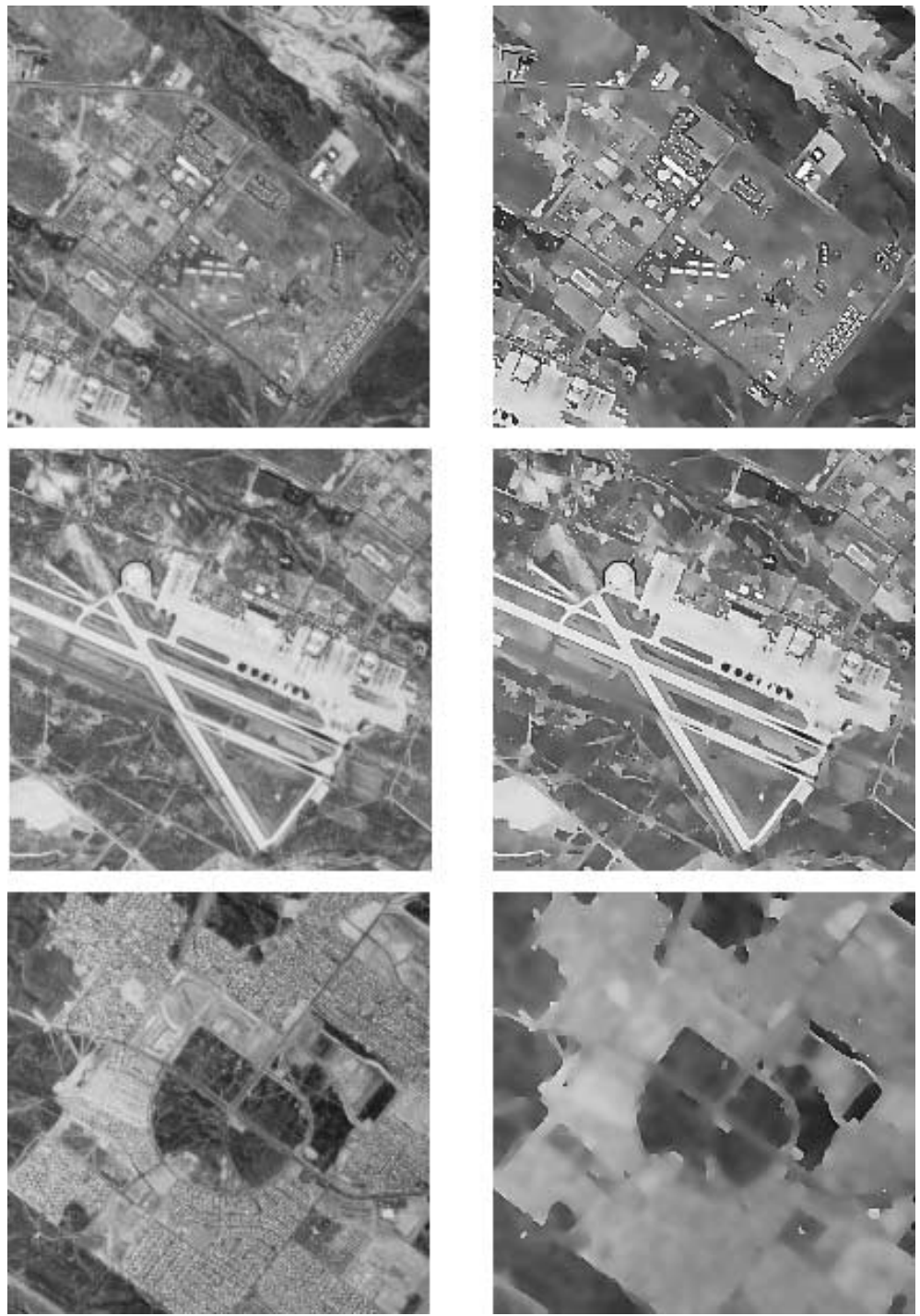

Fig. 7. Illustration of the efficiency of the new filtering scheme. Left column: multispectral satellite images, besides images processed with the new method 

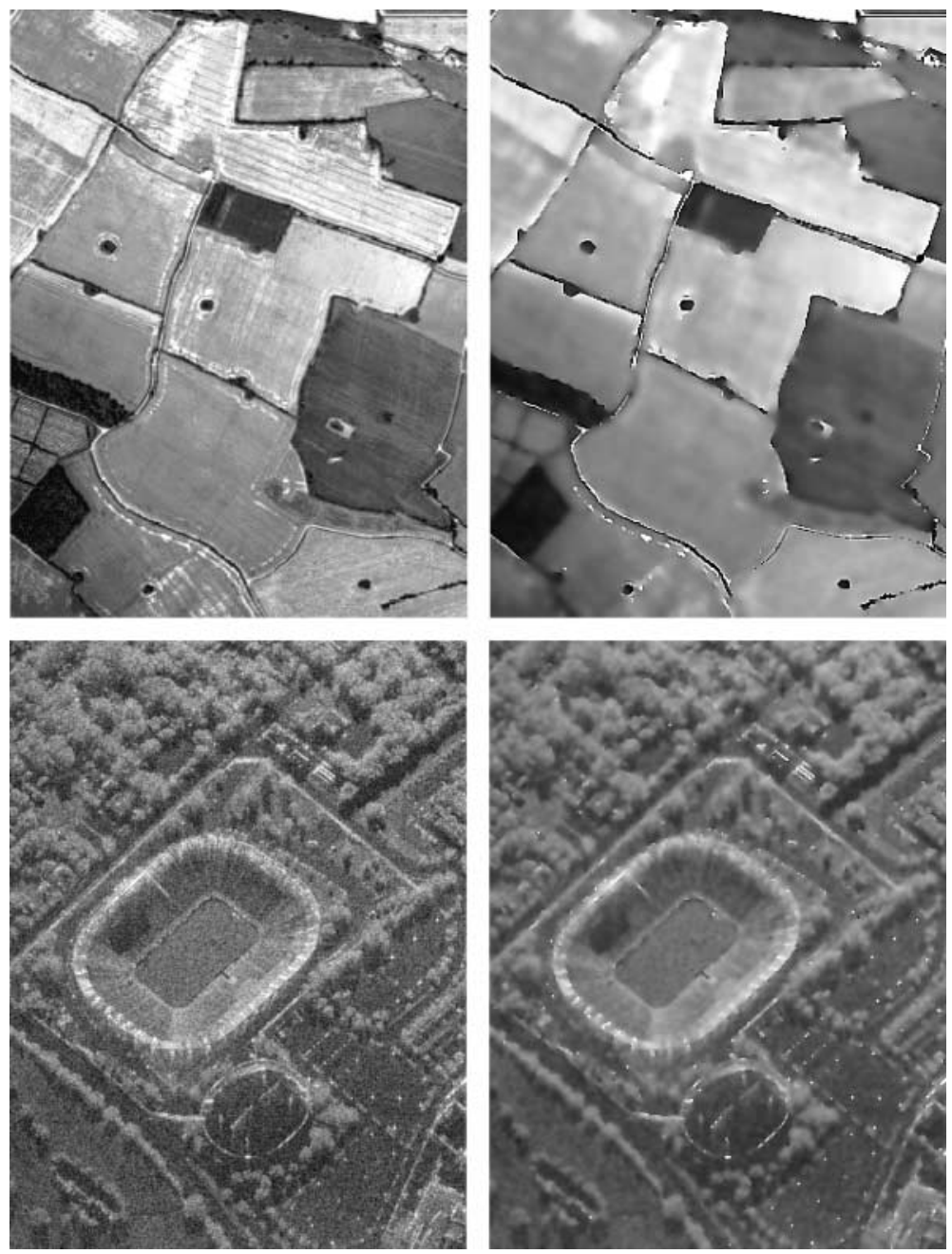

Fig. 8. Illustration of the efficiency of the new combined forward and backward anisotropic diffusion scheme. Left: color aerial image and below a test SAR image, to the right images enhanced with the new filtering scheme presented in the paper 